\title{
Thermal design of the SCUBA-2 instrument detector stage and enclosure
}

\author{
Adam L. Woodcraft ${ }^{*} a$, Fred C. Gannaway ${ }^{a}$, David C. Gostick ${ }^{b}$ and Dan Bintley ${ }^{a}$ \\ ${ }^{a}$ School of Physics and Astronomy, University of Wales, Cardiff, P.O. Box 913, Cardiff, CF24 3YB, \\ UK \\ ${ }^{b}$ UK Astronomy Technology Centre, Royal Observatory, Edinburgh, Blackford Hill, Edinburgh, EH9 \\ 3HJ, UK
}

\begin{abstract}
The SCUBA-2 instrument is a new wide field submillimeter imager currently being designed for the James Clerk Maxwell telescope on Mauna Kea in Hawaii. The instrument will observe simultaneously in the 450 and $850 \mu \mathrm{m}$ bands and has a field of view of approximately 50 square arcminutes. To meet the performance requirements the detectors require a heat sink at a temperature of $50 \mathrm{mK}$ or lower, and must be surrounded by an enclosure at a temperature of $1.1 \mathrm{~K}$ or below. Cooling is provided by the mixing chamber and still of a cryogen-free dilution refrigerator (DR), via thermal links of the order of a metre in length. A challenging set of requirements result from the need for a small temperature drop between the detectors and the refrigerator insert despite the large distance between them, the need to provide flexibility in the links to allow for movement during thermal contraction, and the need to allow for the detectors to be removed from the cryostat. Further, the arrays require a mounting structure which provides rigid mechanical support from the 1-K stage yet causes a very small heat input to millikelvin stage. This paper describes the design which has been evolved to meet these difficult (and often conflicting) requirements.

Copyright 2004 Society of Photo-Optical Instrumentation Engineers. This paper was published in Astronomical Structures and Mechanisms Technology, Proceedings of the SPIE, Volume 5498, edited by Antebi, Joseph; Lemke, Dietrich, pp. 446-454 (2004) and is made available as an electronic reprint with permission of SPIE. One print or electronic copy may be made for personal use only. Systematic or multiple reproduction, distribution to multiple locations via electronic or other means, duplication of any material in this paper for a fee or for commercial purposes, or modification of the content of the paper are prohibited.
\end{abstract}

Keywords: SCUBA-2, submm, thermal design, millikelvin

\section{INTRODUCTION}

The SCUBA-2 instrument ${ }^{1}$ is a new wide field submillimeter imager currently being designed for the James Clerk Maxwell telescope on Mauna Kea in Hawaii. The instrument will observe simultaneously in the 450 and $850 \mu \mathrm{m}$ bands and has a field of view of approximately 50 square arcminutes. To meet the performance requirements the detectors require a heat sink at a temperature of $50 \mathrm{mK}$ or lower, and must be surrounded by an enclosure (the " $1-\mathrm{K}$ box") at a temperature of approximately $1 \mathrm{~K}$. Cooling is provided by the mixing chamber and still of a cryogen-free dilution refrigerator (DR); this is located at a considerable distance from the detectors.

Thermal links are therefore required from the dilution refrigerator insert to the detectors and 1-K box. These links must be flexible to allow for relative motion during thermal contraction. They must also contain demountable joints so that components can be removed for repair or replacement. The number of such joints needs to be kept to a minimum since every time a joint is broken and made there is the chance of wear or damage, reducing thermal performance. It is also necessary to provide a support for the detectors which mounts them rigidly to the 1-K box while minimizing the heat input to the detector stage from the $1-\mathrm{K}$ box. The $1-\mathrm{K}$ stage must completely surround the detectors to prevent radiation and straylight reaching them from higher temperatures. This paper describes the design for the millikelvin and 1-K stages which has been evolved to meet the above requirements, and explains the choices made. The overall design of the instrument is described elsewhere. ${ }^{2}$ 


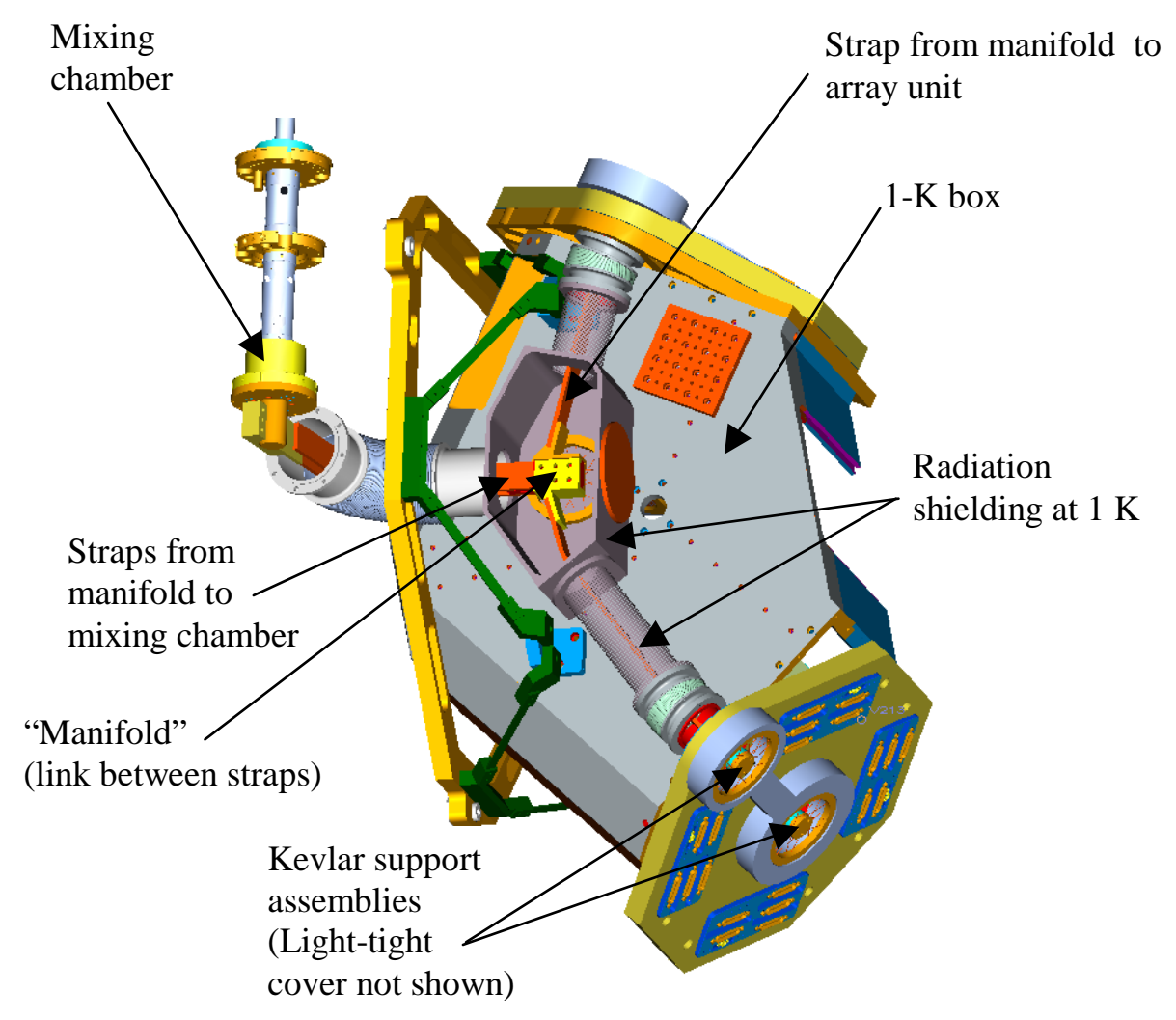

Figure 1. Overview of 1-K box and detector units. Note that the 1-K thermal strap system is not shown in this Figure.

\section{OVERVIEW}

Figure 1 shows an overview of the $1-\mathrm{K}$ box and related components. There are two detector arrays, operating at wavelengths of $450 \mu \mathrm{m}$ and $850 \mu \mathrm{m}$. Each array consists of a mosaic of four sub-arrays. The sub-arrays form part of a sub-array unit which consists of a detector assembly operating at temperatures below $100 \mathrm{mK}$, and a SQUID electronics board operating at approximately $1 \mathrm{~K}$; the two sections are linked by a flexible ribbon cable - see Figs 2 and 3. The SQUIDs are operated at $1 \mathrm{~K}$ since the heat dissipated is too large for the millikelvin stage to accommodate. The four sub-array units for each array are mounted in a detector unit (also referred to as a focal plane unit); this provides heat sinking at the appropriate temperatures, and can be removed from the 1-K box. It also provides mechanical support between the two different temperature sections of each sub-array unit; providing sufficiently rigid support while reducing the heat transferred from the $1-\mathrm{K}$ to the $\mathrm{mK}$ stage requires very careful design.

The 1-K box is manufactured from aluminium alloy (6082 T6), and provides mechanical support and magnetic and straylight shielding for the detectors and optical components. Aluminium was chosen since it has a low density and is easy to machine. A beam splitter (dichroic) inside the $1-\mathrm{K}$ box sends the incoming optical beam to the two detector arrays. The box is cooled by a thermal link to the dilution refrigerator still. To avoid the poor thermal contact which is likely for a bare aluminium to copper joint, thermal contact is made via a large area epoxy filled joint made between a copper plate and the aluminium box. Further copper straps run from this copper plate to the 1-K section of the detector units to give better thermal contact than would be achieved by relying on conduction through the 1-K box. A demountable bolted joint allows the $1-\mathrm{K}$ box to be removed from the cryostat.

To provide uniform thermal contact to the silicon detectors without causing damage due to differential thermal contraction, the detector heat sinks consist of many thin beryllium copper tines individually bonded to the detectors. ${ }^{3}$ The heat sink is thus referred to as the "hairbrush". To mount a sub-array unit in a detector unit, the hairbrush (with the detector

\footnotetext{
*Adam.Woodcraft@physics.org; phone +44 8707651873
} 


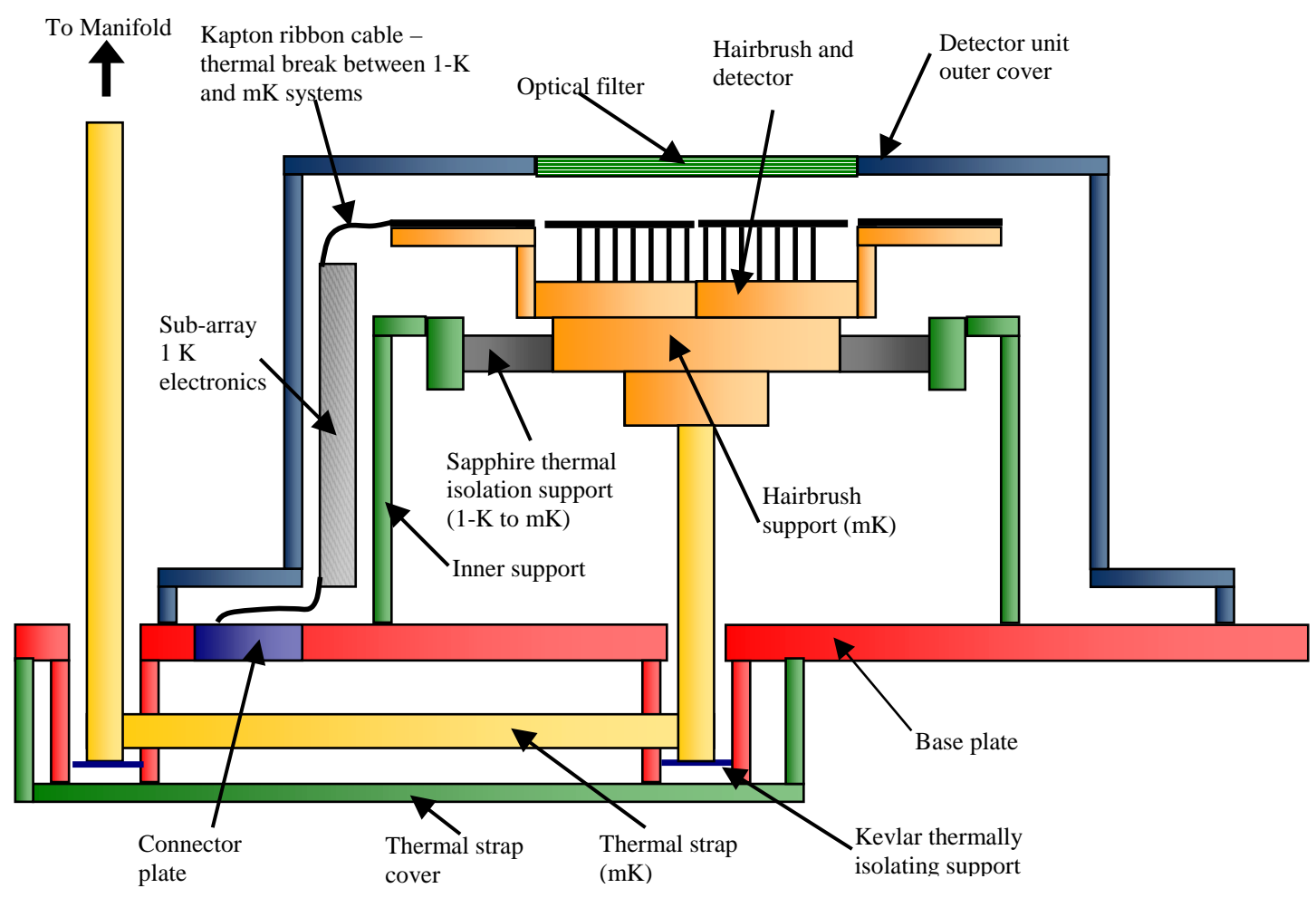

Figure 2. Schematic of a detector unit, showing two of the four subarray units.

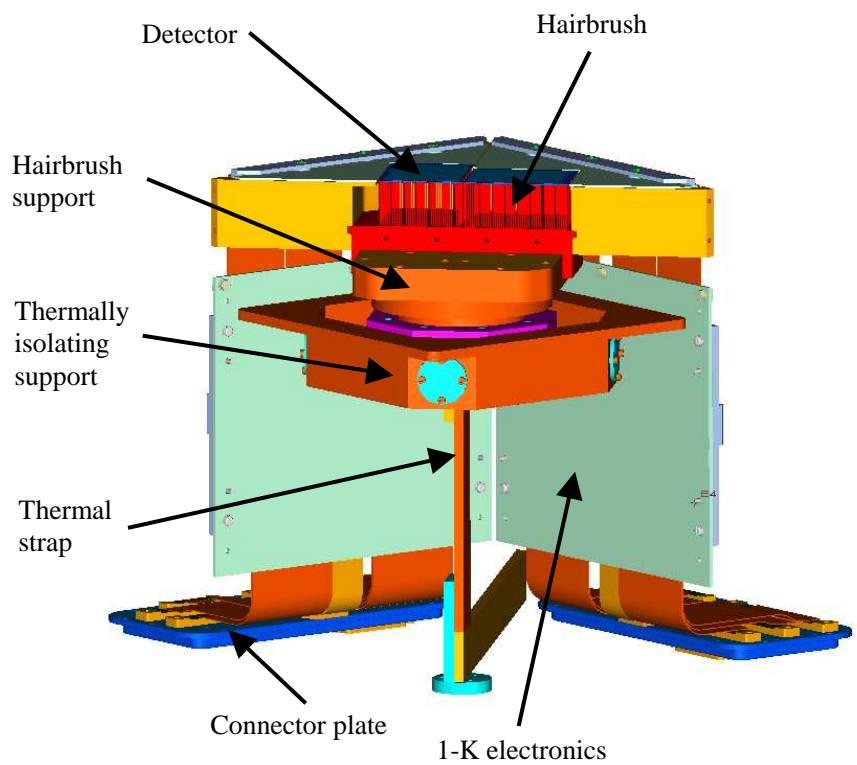

Figure 3. Image of detector unit; for clarity, some components are not shown. Only two sub-array units are shown in place. 


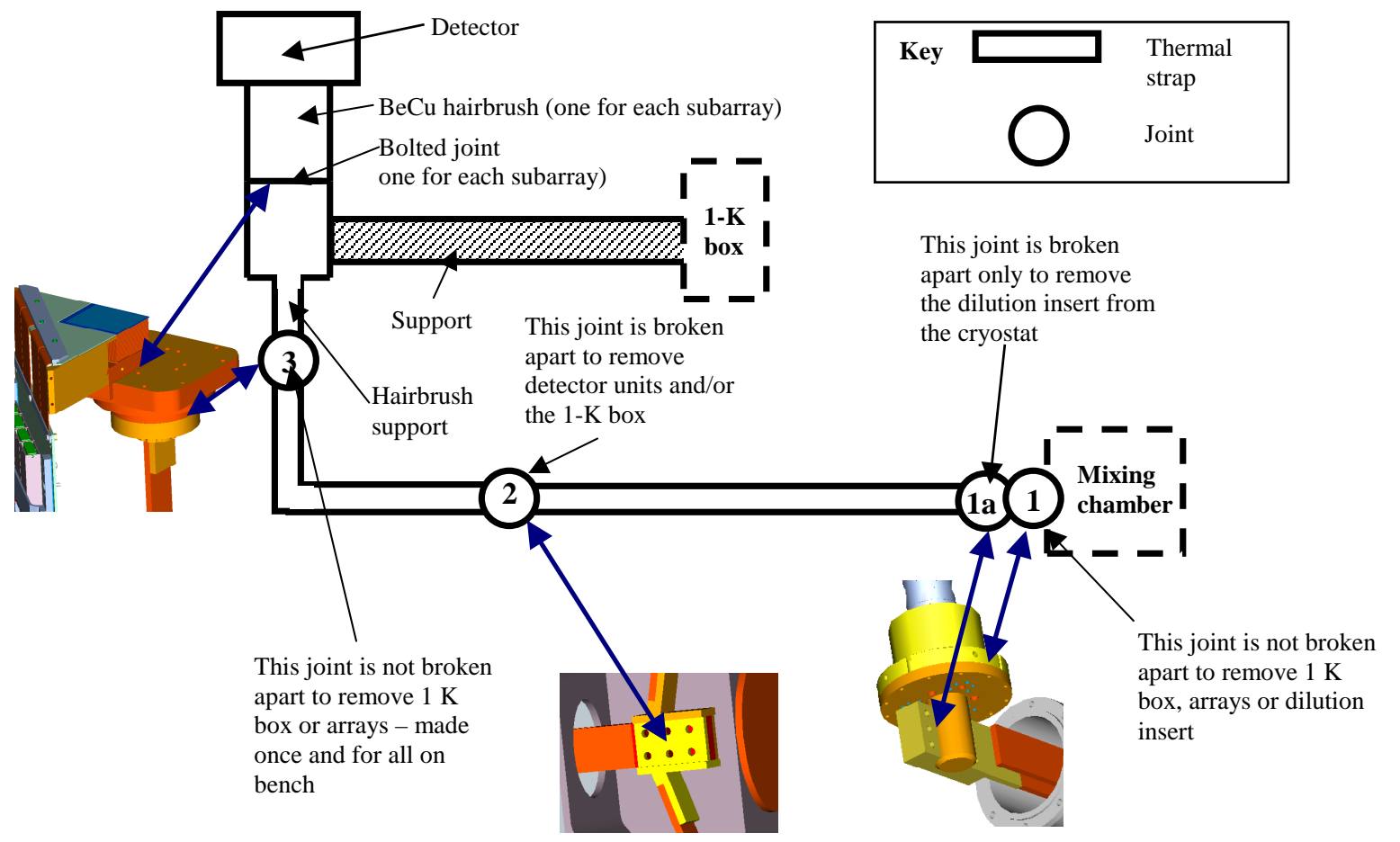

Figure 4. Schematic of the thermal design of the millikelvin system. The entire system is duplicated for the two arrays. The joint numbers refer to numbers used in the text.

already attached) is bolted to a copper mount (the hairbrush support), which is itself supported from the 1-K structure. Straps link the hairbrush supports for the two arrays to the dilution refrigerator mixing chamber. Demountable joints in these straps allow the detector units to be removed from the 1-K box, and the 1-K box and DR insert to be removed from the cryostat. The entire system at millikelvin temperatures is surrounded by a radiation shield at approximately $1 \mathrm{~K}$.

\section{MILLIKELVIN THERMAL LINKS}

Figure 4 shows a schematic of the layout of the millikelvin thermal system. The thermal links are made from annealed $5 \mathrm{~N}$ purity copper $0.1 \mathrm{~mm}$ thick foil; annealing and using such high purity copper provides improved thermal conductivity compared with using standard commercial copper, while using thin foils provides flexibility to allow for movement during thermal contraction. Each strap consists of a stack of 50 foils, electron beam welded into commercial (electrolytic tough pitch) copper end pieces; using commercial copper for these components is cheaper than buying $5 \mathrm{~N}$ purity bulk copper and has a small impact on overall conductivity. The thermal conductance across e-beam welded joints is similar to that through bulk material. Demountable contacts are made using bolted joints between gold plated commercial copper surfaces; the gold plating prevents the formation of the oxide layer which would form on bare copper surfaces and degrade the thermal conductance across the joint. ${ }^{4}$ Spring (Belleville) washers are used on the bolts to maintain pressure as the joint is cooled down. Since copper is easily deformed, in order to be able to repeatedly make the joints in a reliable fashion, the bolts are screwed into stainless steel washers rather than directly into the copper parts.

A plate with a protruding cylinder (Figure 4, joint 1) is permanently bolted to the dilution refrigerator mixing chamber. The end pieces of the two thermal straps are bolted to each other around the cylinder (joint 1a). This joint has to be broken apart only if the DR insert needs to be removed from the cryostat for repair. The advantage of this joint geometry is that good contact can be made without accurately choosing a particular orientation of the mixing chamber to match the direction taken by the straps. For each array, a strap made from single lengths of foil runs to joint 2 (also known as the "manifold"). This joint is made by bolting flat copper surfaces together, and will be broken apart to remove detector units or the entire $1-\mathrm{K}$ box from the cryostat. 


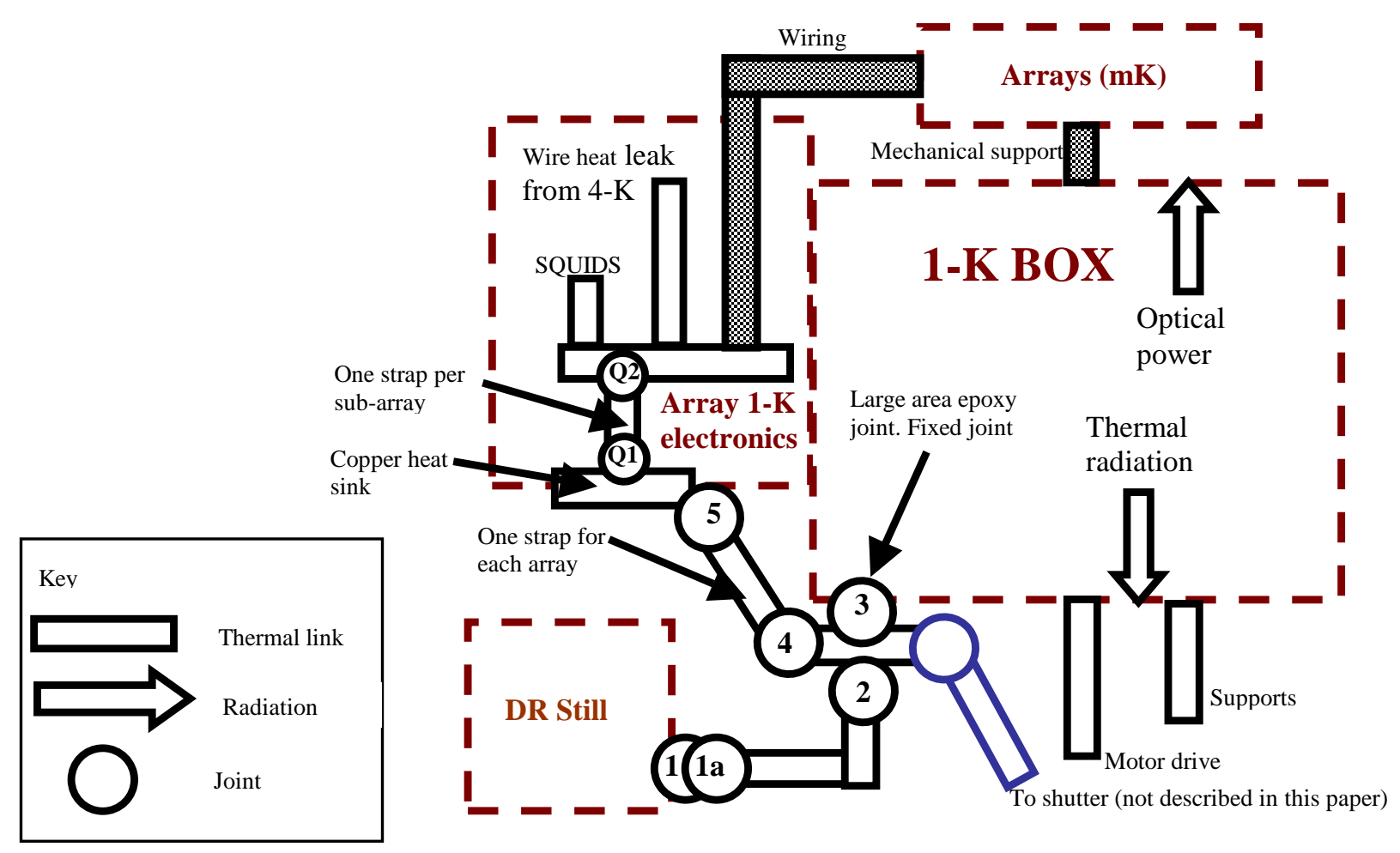

Figure 5. Schematic of the $1-\mathrm{K}$ thermal system. The joint numbers refer to numbers used in the text.

Beyond this joint, a "U" shaped strap runs to the detectors. This is made out of three sections of foil straps, joined by electron beam welds to commercial copper corner pieces. The corner pieces also provide a location to mount supports

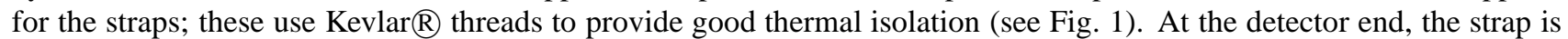
electron beam welded to a circular end piece; this is bolted to the array support (joint 3 ). This joint is required for ease of fabrication; it can be made using large torques before integrating the strap with the instrument. It is not intended that this joint is ever broken apart once made. Figure 1 shows the straps in place in the instrument.

The sub-array units are mounted to the hairbrush support by bolted joints; these joints need to be broken apart to remove an individual sub-array unit. The detector heat sinks (hairbrushes) are made from gold plated beryllium copper; these joints therefore differ from the other bolted joints which are made between copper surfaces.

The millikelvin straps are enclosed in radiation shielding maintained at approximately $1 \mathrm{~K}$. This provides thermal radiation and straylight protection for the detectors, and is a more reliable solution than providing baffling where the millikelvin straps enter the detector units. Light baffles would require parts which were very closely fitting but did not touch since a touch would cause a thermal short from the millikelvin to the $1-\mathrm{K}$ system.

\section{1-K THERMAL LINKS}

Various thermal links are used to maintain components at a temperature of approximately $1 \mathrm{~K}$, using cooling power from the still of the dilution refrigerator - see Fig. 5. The links are made using $5 \mathrm{~N}$ purity foil with commercial copper end pieces in the same manner as the millikelvin straps (see Sect. 3).

The 1-K box is cooled by a thermal link running to the dilution refrigerator still. It is very difficult to make good thermal contact to aluminium (either pure or as an alloy), due to the ready formation of an oxide layer. While in principle gold plating offers a solution, in practice it is very hard to gold plate aluminium reliably, and without trapping oxide under the plating. In addition, a joint between two dissimilar materials such as copper and aluminium may have reliability problems due to differential contraction during thermal cycling. 

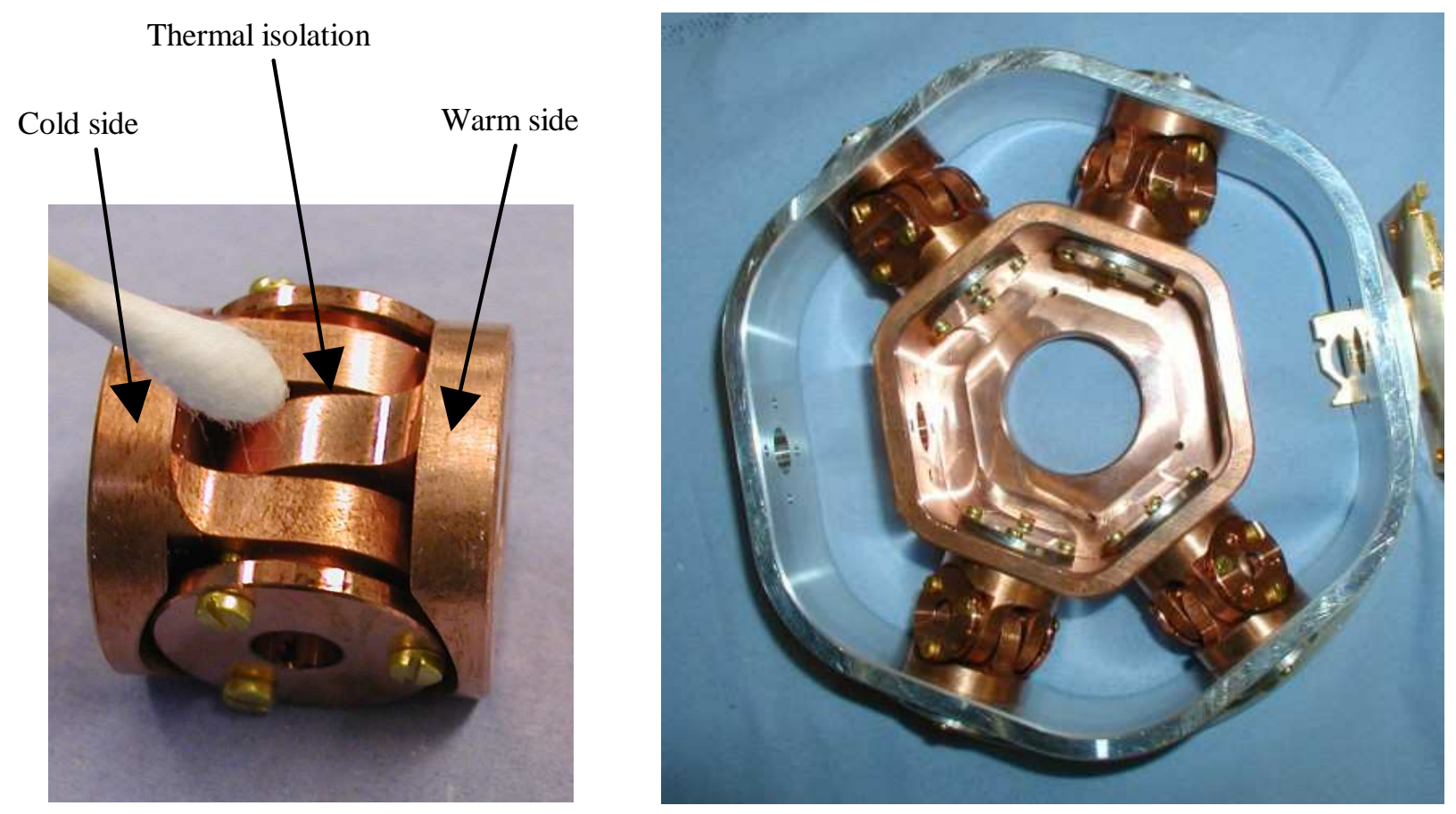

Figure 6. Left: a single sapphire thermal isolation joint. Right: the prototype thermal isolation structure.

We have therefore chosen to make a joint between the side of the box and a copper plate using epoxy* (Fig. 5, joint 3 ). While the thermal conduction through epoxy is poorer at low temperatures than for good metal to metal contact, the conduction is adequate and predictable, and likely to be much better than a joint between copper and aluminium. Such a joint has a much larger effective contact area than a metal to metal joint, since the epoxy will fill irregularities in the two surfaces being joined, whereas two metal surfaces will touch only at a small number of raised points. The joint is, of course, not demountable; a conventional bolted gold plated copper to copper joint is therefore used to attach the thermal link to the still (joint 2). This joint is broken apart to remove the 1-K box from the SCUBA-2 cryostat.

The copper plate is also used as a mounting point for straps running to the detector units. These are used to provide an all-copper path for cooling the sub-array unit 1-K electronics (SQUID) stages, as well as the 1-K heat sinks for the detector wiring. Straps run to both of the detector units; the straps then branch to link to copper plates at the base of each sub-array unit (the connector plates). Copper straps internal to the sub-array units link the copper plates to the circuit boards housing readout electronics (SQUIDS) to remove the dissipated heat.

A further strap, ${ }^{2}$ attached to the epoxy joint copper plate, is used to provide cooling to the SCUBA-2 shutter. ${ }^{1}$

\section{THERMAL ISOLATION}

Maintaining good thermal isolation between the millikelvin and 1-K stages is crucial to the successful operation of the instrument. There are two main paths for heat flow between these two stages: the mechanical support for the arrays (and thermal straps), and the array wiring. The heat leak down the array wiring is minimized by using a different type of wiring to the rest of the instrument, consisting of niobium tracks sputtered onto a flexible Kapton $\mathbb{R}$ membrane. Kapton is a good insulator at low temperatures, and this design enables the cross section of the wires themselves to be minimized.

The arrays are supported using a rigid structure. This provides thermal isolation by utilising the poor thermal conductance across a joint between sapphire discs with alumina powder sandwiched between them. ${ }^{5}$ Good thermal isolation is achieved because the two sapphire surfaces only touch at a small number of points, giving a very small true contact area.

${ }^{*}$ Stycast $2850 \mathrm{FT}$ 


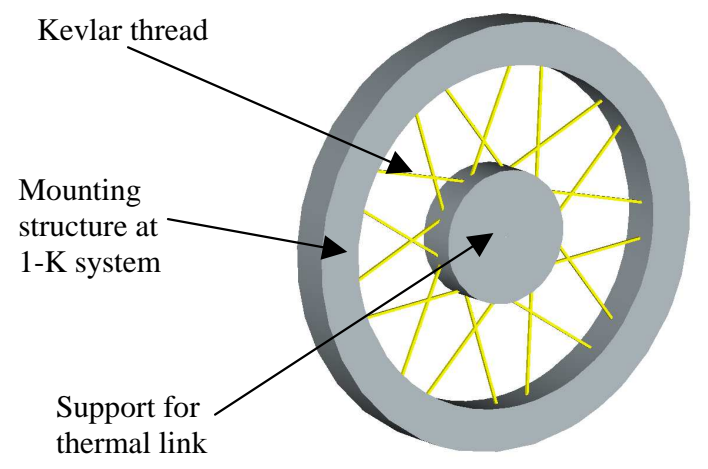

Figure 7. Kevlar structure used to support the millikelvin straps.

A hard material such as sapphire is required since softer materials would deform to some extent, increasing the contact area. The powder decreases the contact area further from that of two sapphire surfaces in direct contact. This provides a sufficiently low thermal load while supporting the arrays without the risk of significant motion during cool-down. The open structure allows access to the back of the arrays, enabling the use of bolted joints between the hairbrush supports and hairbrushes. Tests have determined a suitable design for a single sapphire-powder-sapphire joint, and a support structure has been designed which uses four of these joints to provide a support which is constrained in all three dimensions. A prototype system has been constructed (Fig. 6); the thermal performance has been measured at the appropriate temperatures, and the mechanical strength has been measured at room temperature. The mechanical performance should not be significantly different at lower temperatures.

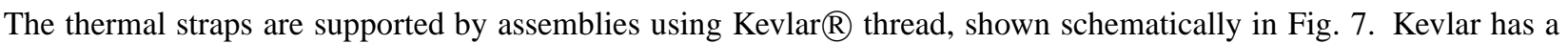
low thermal conductivity at low temperatures. Moreover it has a high tensile strength, so that a small total cross-section is required for a mechanical support. These two properties enable supports to be made with very low thermal conductance. ${ }^{6-8}$ However, Kevlar suffers from creep over time, and expands as the temperature is reduced. Therefore if precise positioning is necessary, elaborate tensioning systems are required. For this reason, the sapphire isolation stage was chosen as a simpler and more robust solution to support the arrays, where accurate positioning is critical.

\section{THERMAL MODELLING AND REQUIREMENTS}

Thermal models have been used to confirm that this design meets the requirements, and to identify critical areas. Where possible, parameters used in the model are based on reliable data from the literature. Where appropriate values could not be found in the literature, measurements were carried out on representative samples. Measurements were necessary for the conductance across the copper to beryllium copper joint between the hairbrushes and the hairbrush supports, across the sapphire isolation stage and along the CFRP (carbon fibre reinforced plastic) struts linking the 1-K box with components at higher temperatures. ${ }^{2}$ While values are available in the literature for the conductivity of CFRP, the range of values

Table 1. Power loading on the detector (millikelvin) stage. This table gives figures for the $450 \mu \mathrm{m}$ array, assuming a temperature of $1.1 \mathrm{~K}$ for the $1-\mathrm{K}$ stage. The values for the $850 \mu \mathrm{m}$ stage are slightly lower, due to different values for the power dissipated by the detectors.

\begin{tabular}{lc}
\hline Source & Power $(\mu \mathrm{W})$ \\
\hline Detectors & 3.9 \\
Sapphire thermal isolation support & 2.5 \\
Kevlar@ thermal strap supports & 0.25 \\
Array wiring & 6.1 \\
Radiation & $7.1 \times 10^{-5}$ \\
\hline
\end{tabular}


Table 2. Temperature profile along the thermal straps to the $450 \mu \mathrm{m}$ detector. The power column shows the thermal power through each component. Note that the power is lower for the last two entries because there are four hairbrushes for each array, and the heat through the array supports does not pass through the hairbrushes.

\begin{tabular}{lccc}
\hline Component & Temperature drop $(\mathrm{mK})$ & Temperature at warm end $(\mathrm{mK})$ & Power $\mu \mathrm{W}$ \\
\hline Mixing chamber & - & 31.5 & 12.7 \\
Joint 1 & 0.9 & 32.4 & 12.7 \\
Joint 1a & 0.9 & 33.3 & 12.7 \\
Strap & 1.0 & 34.3 & 12.7 \\
Joint 2 & 0.9 & 35.2 & 12.7 \\
Strap & 1.8 & 37.0 & 12.7 \\
Joint 3 & 0.8 & 37.8 & 12.7 \\
Hairbrush support & 0.1 & 37.9 & 12.7 \\
Hairbrush joint & 0.2 & 38.1 & 2.5 \\
Hairbrush & 0.0 & 38.1 & 2.5 \\
\hline
\end{tabular}

Table 3. Power loading on the 1-K stage.

\begin{tabular}{lc}
\hline Source & Power $(\mathrm{mW})$ \\
\hline Radiation & $8.66 \times 10^{-3}$ \\
Optical radiation & $0.07 \times 10^{-3}$ \\
Mechanical supports & 0.210 \\
Array wiring & 0.515 \\
Shutter & 0.045 \\
1-K electronics & 0.320 \\
\hline
\end{tabular}

Table 4. Temperature profile through the $1-\mathrm{K}$ system. Note that from joint 2 there are two paths; into and through the 1-K box, and along the straps to the sub-arrays. The decrease in power values as each system is followed is due to the fact that the straps branch into two to reach the two arrays; each strap then branches into four straps; one to each sub-array.

\begin{tabular}{lccc}
\hline Component & Temperature drop $(\mathrm{mK})$ & Temperature at warm end $(\mathrm{mK})$ & Power mW \\
\hline Still & - & 900.0 & 1.10 \\
Joint 1 & 2.8 & 902.8 & 1.10 \\
Joint 1a & 2.9 & 905.7 & 1.10 \\
Strap & 3.6 & 909.3 & 1.10 \\
Joint 2 & 2.8 & 912.1 & 1.10 \\
Joint 3 & 1.3 & 913.4 & 0.22 \\
Through box & 3.3 & 916.7 & 0.22 \\
\hline Joint 2 & - & 912.1 & 0.44 \\
Joint 4 & 5.6 & 917.7 & 0.44 \\
Strap & 7.3 & 925.0 & 0.44 \\
Joint 5 & 5.6 & 930.6 & 0.44 \\
Joint Q1 & 1.4 & 932.0 & 0.11 \\
Strap & 3.0 & 935.0 & 0.11 \\
Joint Q2 & 1.0 & 936.0 & 0.11 \\
\hline
\end{tabular}


for different types of CFRP is large ${ }^{9}$ and no values were available for the type planned. In addition, there is always the possibility of lot-to-lot variations with materials of this type, since the low temperature conductivity is not a parameter of importance to most users of the material. Measurements were therefore made on samples from the actual sheets to be used to make the components in the instrument.

The total power loading on the millikelvin stage is shown in Table 1, and the temperature profile in Table 2. The results meet the goal of a hairbrush temperature below $40 \mathrm{mK}$. The requirement of $50 \mathrm{mK}$ is met even with all the power values in Tabel 1 doubled. Note that the temperature drop between the dilution refrigerator and the hairbrush is not dominated by any one component.

Results from modelling the 1-K system are shown in Tables 3 and 4. Again, the specifications are met with considerable margin; the maximum temperature of $936 \mathrm{mK}$ is a long way below the requirement of $1.1 \mathrm{~K}$; with the power loads in Table 3 doubled, the highest temperature becomes $971 \mathrm{mK}$. As with the millikelvin system, the overall temperature drop is not dominated by any one component. The cool-down times for both systems, predicted by dynamic thermal modelling, are described in Ref. 2.

\section{CONCLUSION}

The requirements for the thermal design for the SCUBA-2 detector stage and enclosure are demanding. Accurate thermal modelling is essential for assurance that the design will operate as planned, and in order to identify any critical areas. The solution presented here is largely based upon established concepts, for which reliable values for thermal modelling are available; this approach minimizes risk. Where it was not possible to do this, prototype components were constructed and tested, and the results used in the models. The results of thermal modelling show that the design should perform to well within the specifications.

\section{ACKNOWLEDGMENTS}

The SCUBA-2 project is funded by the UK Particle Physics and Astronomy Research Council, the JCMT Development Fund and the Canadian Foundation for Innovation.

\section{REFERENCES}

1. M. D. Audley, W. S. Holland, W. D. Duncan, D. Atkinson, M. Cliffe, M. Ellis, X. Gao, D. C. Gostick, T. Hodson, D. Kelly, M. J. MacIntosh, H. McGregor, T. Peacock, I. Robson, I. Smith, K. D. Irwin, G. C. Hilton, J. N. Ullom, A. Walton, C. Dunare, W. Parkes, P. A. R. Ade, D. Bintley, F. Gannaway, M. Griffin, G. Pisano, R. V. Sudiwala, I. Walker, A. Woodcraft, M. Fich, M. Halpern, G. Mitchell, D. Naylor, and P. Bastien, "SCUBA-2: A large-format TES array for submillimetre astronomy," Nuclear Instruments and Methods in Physics Research A 520(1-3), pp. 479-482, 2004.

2. D. Gostick, D. Montgomery, B. Wall, H. McGregor, M. Cliffe, A. Woodcraft, and F. Gannaway, "The cryo-mechanical design of SCUBA-2 - a wide field imager for the James Clerk Maxwell Telescope," in SPIE Vol. 5492, Ground-Based Instrumentation for Astronomy, 2004, submitted.

3. W. Duncan, D. Audley, W. Holland, D. Atkinson, T. Baillie, M. Cliffe, M. Ellis, X. Gao, D. Gostick, T. Hodson, D. Kelley, M. MacIntosh, H. McGregor, T. Peacock, I. Smith, I. Robson, A. Walton, W. Parkes, C. Dunare, S. Smith, J. Terry, T. Stevenson, A. Gundlach, A. Ruthven, P. Ade, C. Tucker, F. Gannaway, I. Walker, R. Sudiwala, A. Woodcraft, M. Griffin, D. Bintley, E. Schulte, M. Fich, J. Kycia, M. Halpern, J. Molnar, B. Burger, D. Naylor, P. Bastien, G. Mitchell, K. Irwin, G. Hilton, J. Ullom, C. Reintsema, L. Vale, L. Ferreira, and W. Doriese, "SCUBA-2 arrays to system interfaces," Nuclear Instruments and Methods in Physics Research A 520(1-3), pp. 427-430, 2004.

4. I. Didschuns, A. L. Woodcraft, D. Bintley, and P. C. Hargrave, "Thermal conductance measurements of bolted copper to copper joints at sub-Kelvin temperatures," Cryogenics 44(5), pp. 293-299.

5. K.-H. Yoo and A. C. Anderson, "Thermal impedance of pressed contacts at temperatures below 4 K," Cryogenics 23(10), pp. 531-2, 1983.

6. P. R. Roach, "Kevlar support for thermal isolation at low temperatures," Review of Scientific Instruments 63(5), pp. 3216-17, 1992.

7. G. Ventura, M. Barucci, E. Gottardi, and I. Peroni, "Low temperature thermal conductivity of Kevlar," Cryogenics 40(7), pp. 489-91, 2000.

8. L. Duband, L. Hui, and A. Lange, "Thermal isolation of large loads at low temperature using Kevlar rope," Cryogenics 33(6), pp. 643-647, 1993.

9. R. P. Reed and M. Golda, "Cryogenic composite supports: a review of strap and strut properties," Cryogenics 37(5), pp. 233-50, 1997. 